P561 DEVELOPING SURVEILLANCE TOOLS TO MEASURE MSM'S HIV INFECTION RISK IN THE ERA OF COMPLEX BIOBEHAVIOURAL PREVENTION STRATEGIES

${ }^{1}$ Jamie Frankis*, ${ }^{2}$ Paul Flowers, ${ }^{3}$ Lesley Wallace, ${ }^{3}$ David Goldberg, ${ }^{4}$ Martin Holt, ${ }^{3}$ Lisa Mcdaid. ${ }^{1}$ Glasgow Caledonian University, School of Health and Life Sciences, Glasgow, UKi ${ }^{2}$ University of Glasgow, MRC/CSO Social and Public Health Sciences Unit, Glasgow, UK ${ }^{3}$ Health Protection Scotland, QU, UK; ${ }^{4}$ UNSW Sydney, Centre for Social Research in Health, Sydney, Australia

\subsection{6/sextrans-2019-sti.635}

Background Behavioural surveillance enables monitoring of disease epidemics, assessment of health promotion, development of health policy and service planning. However, the emergence of multiple effective biobehavioral risk management strategies to prevent HIV transmission (including PrEP, treatment-as-prevention (TasP), negotiated safety (NS), serosorting, condom use), demands new surveillance tools able to reflect this complexity. Here, we critically discuss our new surveillance tool, developed to measure MSM's biobehavioural HIV risk-taking. Methods Items were developed with surveillance experts in Scotland and Australia, piloted within Scotland, then delivered within the Scottish Gay Men's Bar Survey ( $n=972$ MSM), across the commercial gay scenes of Scotland's two largest cities in 2017. Participants were asked about sex with regular and casual partners separately, condom use and condomless anal intercourse (CAI), their HIV status, PrEP use and their partners' HIV and undetectable viral load (UVL) status.

Results Overall, 5.6\% of participants were HIV+. For our HIV-/untested participants, $67.7 \%$ were categorised as 'lower risk' since, in the last year, they reported; (i) PrEP use (4.5\%) (ii) no CAI (37.1\%) (iii) Negotiated safety; CAI with 1 regular HIV- partner (16\%) (iv) TasP; CAI with 1 regular HIV+ UVL partner $(0.4 \%)$ (v) Serosorting; CAI with casual and/or multiple regular HIV- partners (4.1\%) (vi) Both serosorting and TasP CAI with multiple regular and casual partners (5.4\%) The remaining 32.3\% were categorised as 'higher risk'; HIV-/ untested men not on PrEP who reported CAI with HIV status-unknown partners, or HIV+ partners with unknown/ detectable viral load. Of these, $67.6 \%$ report $2+$ CAI partners, making them potentially eligible for PrEP in Scotland.

Conclusion No CAI, NS and PrEP were the key biobehavioural risk strategies used. Two-thirds of our higher risk men could, but are yet to, benefit from PrEP. Although offering new insights, we invite critical engagement with these risk criteria which present different, yet related challenges for researchers and MSM alike.

Disclosure No significant relationships.

\section{P562 HEALTH SEEKING BEHAVIOUR AND ACCEPTABILITY OF ONLINE OUTREACH EFFORTS AMONG MSM USING SEX- SEEKING APPS/WEBSITES}

${ }^{1}$ David Brennan, 'Maya Kesler, ${ }^{2}$ Nathan Lachowsky, ${ }^{1}$ Tsegaye Bekele. 'University of Toronto, Factor-inwentash Faculty of Social Work, Toronto, Canada; ${ }^{2}$ University of Victoria, School of Public Health and Social Policy, Victoria, Canada

10.1136/sextrans-2019-sti.636

Background Gay, bisexual, and other men who have sex with men (GBM) commonly use the Internet to find sexual partners and look for sexual health information. Little is known about online health information seeking behaviour and whether GBM welcome this information on socio-sexual apps websites.

Methods GBM aged 14+ from Ontario were recruited into the \#iCruise study via socio-sexual websites/apps from 07/ 2017-01/2018. Participants reported online health seeking behaviours and acceptability of sexual health information appearing as a clickable link/pop-up on socio-sexual apps/websites. Stratified by HIV status, multivariable logistic regressions were used to determine associations between sociodemographic variables and online health-seeking behaviour.

Results There were 910 GBM who were eligible for this baseline cross-sectional analysis. The majority reported being White (62\%), and gay-identified (65\%). A minority of participants were HIV-positive (12\%), university educated (44\%), and lived in rural areas (12\%). Over two-thirds $(69.3 \%$, $\mathrm{n}=631 / 910)$ reported looking up online health information in the previous 3 months. Among HIV-negative/unknown status participants, online health seeking behaviour was negatively associated with older age $(50+$ vs $<29$, OR:0.35,95\% CI:0.23-0.55) and unknown HIV status (vs HIV-negative) (OR:0.57,95\%CI:0.39-0.84) and positively associated with more education (University vs High School OR:2.49,95\% CI:1.55-4.01). There were no significant associations among HIV-positive GBM. Having health information integrated into sex-seeking apps/websites was endorsed by $79 \%$ (agreed/ strongly agreed). Acceptability via clickable link of health topics within sex-seeking apps/websites was very high: closest HIV/STI testing (96\%); ASO website (93\%); mental health information/resources (91\%); public health/government website (90\%); substance use information/resources (89\%). Acceptability of a pop-up/reminder/notification with sexual health information such as how HIV is spread/how to prevent HIV transmission and safer sex practices to reduce HIV/STI transmission was also very high (86\% and $89 \%$, respectively).

Conclusion Acceptability of HIV and sexual health education information being embedded within dating apps/websites was very high and is currently an underutilized educational platform.

Disclosure No significant relationships.

\section{P563 MSM PREDICTIVE MODELING WITHIN A LARGE, LINKED DATABASE OF LABORATORY, SURVEILLANCE, AND ADMINISTRATIVE HEALTHCARE RECORDS}

${ }^{1}$ Travis Salway, ${ }^{2}$ Zahid Butt, ${ }^{2}$ Stanley Wong, ${ }^{2}$ Carmine Rossi, ${ }^{3}$ Jason Wong, ${ }^{2}$ Amanda Yu, ${ }^{2}$ Maria Alvarez, ${ }^{4}$ Troy Grennan, ${ }^{3}$ Mark Gilbert, ${ }^{2}$ Mel Krajden, ${ }^{2}$ Naveed Janjua. ${ }^{1} B C$ Centre for Disease Control, Vancouver, Canada; ${ }^{2} B C C D C$, Vancouver, Canada; ${ }^{3} B C$ Centre for Disease Control, Clinical Prevention Services, Vancouver, Canada; ${ }^{4}$ British Columbia Center for Disease Control, Vancouver, Canada

\subsection{6/sextrans-2019-sti.637}

Background Enumeration or measurement of populations of men who have sex with men (MSM) is critical to developing and evaluating sexually transmitted and bloodborne infection (STBBI) prevention and treatment programs. However, there is a lack of data sources in which sexual orientation or behaviour is measured. In this study, we present the development and validation of a novel model (i.e., 'computational phenotype') to predict MSM status using multiple data sources.

Methods Three disease case surveillance databases (HIV, hepatitis $\mathrm{B}$ and $\mathrm{C}$, and syphilis), a public health laboratory database (which performs $\geq 95 \%$ of all HIV, hepatitis $C$ and syphilis tests in British Columbia), and five administrative 